\title{
Las nuevas tecnologías y la formación médica continuada a distancia
}

\author{
The new technologies and the distance continuing medical education
}

\author{
Helena Segura Badia
}

Consejo Catalán de la Formación Médica Continuada

Tras analizar las ventajas e inconvenientes de las FMC a distancia, se contemplan las tres tipologías básicas: provisión de contenidos (enfoque tradicional), gestión de contenidos (enfoque tradicional con las nuevas tecnologías) y "on-line" (procesos de aprendizaje y comunicación).

Entre los elementos que definen la calidad de los programas formativos a distancia se destacan los tecnológicos, metodológicos y evaluativos. Entre los primeros se incluyen las plataformas, los estándares y la estructura del programa; entre los segundos, los tutores, el diseño de los contenidos , los sistemas de comunicación y las herramientas para crear actividades; y, finalmente, entre los evaluativos, destacan las tipologías destinadas a la evaluación de contenidos, de aprendizaje y de práctica.

Palabras clave: Formación médica continuada a distancia, nuevas tecnologías educativas, e-learning

Correspondencia:

Helena Segura i Badia, Secretaria Técnica

Consejo Catalán de la Formación Médica Continuada

Córcega, 257 pral, Barcelona

E-mail: hsegurabadia@comb.es
The advantages and disadvantages of the distance CME are considered, analyzing the three fundamental typologies: contents' offer (traditional approach,), contents' management (traditional approach plus new technologies) and "on-line" offer (learning processes and communication).

Among the elements related to the quality of the distance CME programmes one can identify the following three essentials: technology, methodology and evaluation.

Technology's elements include the platforms, standards and programme's structure. The tutors, contents' design, communication systems and tools to generate activities are the fundamental elements of the methodologies. Finally, among the evaluation one can identify the different procedures aimed to assess the contents, the learning and the practice (performance).

Key words: Distance CME, new educational technologies, e-learning

\section{INTRODUCCIÓN}

Vivimos en unos momentos de grandes cambios donde la formación a distancia evoluciona al mismo tiempo que se desarrollan las tecnologías de la comunicación y de la información. Las necesidades formativas se incrementan y diversifican y, la inmediatez y la actualización continuada se convierten en factores esenciales 
El siglo XXI pone en crisis a los sistemas de aprendizaje tradicionales, por los cambios sociales y tecnológicos en las universidades, y la tecnología, como herramienta, se convierte en un soporte sustancial al sistema de formación que ofrece la posibilidad de formarse a través de la sociedad del conocimiento, en un mundo, donde todos podemos participar conectándonos a través de la red. Hoy, la oferta formativa se micro especializa al mismo tiempo que aparecen los metacampus virtuales. Estos cambios generales, implican cambios en la educación y hacen que aparezca el paradigma de formarse a lo largo de toda la vida, así como de nuevos conceptos, hoy relacionados con la formación, tales como la ubicuidad (la formación siempre presente) y la holoconectividad (el campus siempre con nosotros) que nos permiten las nuevas tecnologías inalámbricas, y nos abren la posibilidad de poder aprender cuando queramos y, en el momento que queramos, en un mundo lleno de conocimientos accesibles desde cualquier lugar.

Encontramos ya, facultades de medicina virtuales, con programas de convergencia ${ }^{1}$ con sistemas de créditos acumulativos o de transferencia, y con programas, asignaturas con plataformas estandarizadas y intercambiables. La movilidad virtual es el futuro de la formación a distancia que tiene que responder a las nuevas necesidades del profesional a un entorno que le exige una adaptación permanente. Esta necesidad continua de actualización de conocimientos, habilidades y actitudes, va a definir los nuevos modelos educativos en formación continuada, generándonos un cambio de perspectiva y potenciando de forma exponencial la formación on-line, que permite formarse en una dirección más individual y específica, más deacorde a las necesidades del profesional de hoy.

\section{VENTAJAS E INCONVENIENTES}

La formación a distancia puede ofrecer al médico en activo una serie de ventajas: como su aplicación en el lugar de trabajo, la inmediatez de respuesta, la flexibilidad horaria y espacial, la concreción, la personalización, la aplicabilidad, y probablemente pueda responder de manera más rápida y efectiva que otros sistemas tradicionales, con sus limitaciones de tamaño de grupo, horario, burocracia y geografía.

Aún así no podemos decir que la mayoría de los enfoques utilizados sean totalmente satisfactorios, los altos niveles de abandono son muestra de ello, la desmotivación, la sensación de aislamiento por parte de los participantes, la escasa o nula interacción entre profesor-estudiante o entre estudiantes, los resultados en la adquisición de prácticas o habilidades, son aspectos que parecían no poder resolverse. Por ello, en la actualidad, se están desarrollando nuevas estrategias que superen estos inconvenientes.

Cuando, no hace más de una década, apareció el elearning parecía que sustituiría a la formación tradicional, y se abría un nuevo panorama para la formación. Diez años después, el tiempo demostró que, todo y haberse convertido en una herramienta muy significativa, no ha sido ni mucho menos sustitutiva, y que ni todas las acciones formativas deben ni se pueden realizar a través del e-learning.

Cabe tener en cuenta que dependiendo de los objetivos de la actividad, cada uno de los formatos educativos adquiere validez. En la formación tradicional, las aulas ofrecen un aprendizaje formal y práctico; las bibliotecas, el autoaprendizaje, y los trabajos de grupo una formación más colaborativa. Así pues si las clases presenciales son muy útiles para adquirir prácticas concretas, la formación a distancia puede ser muy útil para actualizaciones en las que la inmediatez es un factor importante, y el elearning nos incorpora la posibilidad de establecimiento de redes profesionales de intercambio de conocimientos.

Uno de los principales retos de la formación continuada en el contexto médico es lograr compaginar las necesidades formativas con las exigencias de la actividad diaria y muy importante la posibilidad de realizar actividades muy específicas y muy adaptadas a las necesidades y a la práctica del profesional. La formación a distancia puede adaptarse mejor a estas necesidades, que la formación tradicional.

\section{TIPOLOGÍAS}

Agrupamos las actividades de formación a distancia en tres grandes categorías que se corresponden a las etapas evolutivas de la propia formación:

1․ Provisión de contenidos: reproduce los enfoques tradicionales, basados en el estudio personal de materiales formativos enviados por correo. Con el tiempo y la introducción de nuevas tecnologías las únicas diferencias introducidas fueron que se entregaban los materiales con más agilidad (revistas, monografías, libros,...)

$2^{a}$. Gestión de contenidos: reconoce que la educación es más que una distribución de contenidos. Con la aparición de las tecnologías, se busca, a través de 
estas, reproducir el entorno tradicional, intentando recrear el aula, la experiencia formal (CD, videos, DVD, aulas virtuales,...)

$3^{\circ}$ On-line: buscan reproducir los procesos involucrados en el aprendizaje, y reconocen la necesidad de un elemento clave, la comunicación. Se parte de la idea que un aprendizaje efectivo necesita dialogo, conversación, discusión y reflexión, y los establece como centro de la experiencia del proceso formativo que se concibe como un proceso social, no solitario ${ }^{2}$.

Muchos de los productos de formación on-line que se ofertan se han diseñado principalmente para el autoaprendizaje. Aunque exista la posibilidad de cierta interacción con el tutor, siguen pareciendo meras publicaciones con herramientas tecnológicas añadidas.

Un entorno virtual, bien enfocado, permite realizar actividades de dialogo difícilmente viables en una clase presencial, fomentar el pensamiento crítico por parte del alumno y establecer una Comunidad de Práctica ${ }^{3}$, introduciendo un nuevo elemento, la responsabilidad compartida, donde todos los miembros son partícipes en el proceso de aprendizaje.

En este tipo de formación, es importante evitar la pasividad o no participación, posible en otros enfoques de formación. Para incorporarse a una comunidad de práctica no basta con saber cómo es, sino que el alumno debe saber participar de lleno en las actividades de esta ${ }^{2}$. El e-learning permite ensayar esta participación mientras se van adquiriendo los conocimientos.

El modelo de comunidad de aprendizaje o de práctica, difícilmente aplicable en la formación tradicional gana terreno en el contexto del e-learning, intentando establecer un puente entre la formación y el mundo real. Aún así, quedan muchas cuestiones sobre la calidad y la evaluación de los resultados de este tipo de formación en la práctica real y si bien la idea tiene un gran potencial para el desarrollo de un e-learning más efectivo, es necesario continuar avanzando en esta dirección.

\section{ELEMENTOS DIFERENCIALES QUE DEFINEN LA CALIDAD EN LOS PROGRAMAS DE FORMACIÓN A DISTANCIA}

Nos centraremos en definir aquellos criterios de calidad de la formación a distancia que caracterizan los procesos formativos eficientes ${ }^{4}$. Los agrupamos en tres grandes bloques: Tecnológicos, Metodológicos y Evaluativos.

\section{TECNOLÓGICOS}

La tecnología es el soporte de la formación a distancia, colaborando activa y determinantemente en la obtención de resultados. Unos de los aspectos claves serían los recursos disponibles que han de valorarse no en la calidad de estos, en sí mismos, sino con su pertinencia con los objetivos de la actividad formativa. No es mejor aquella actividad que dispone de más recursos, sino aquella que sus recursos son más idóneos para la consecución de los objetivos.

El proveedor de actividades formativas ha de tener en cuenta que la utilización de los recursos multimedia ha de fomentar el aprendizaje. Demasiadas veces sucede que el elemento innovador tecnológico se convierte en protagonista. La tecnología, es la que debe estar al servicio de la formación.

\section{Plataformas}

Cualquier plataforma educativa ha de garantizar el cumplimiento de los estándares reconocidos, que garantizen la funcionalidad y operabilidad de la estructura educativa.

En un inicio, las estructuras tecnológicas de soporte a estructuras educativas usaban un lenguaje CBT o CAI (Computer Based Training/Computer Arded Instruction) pero fueron evolucionado con internet. La tendencia actual, ya no es la de desarrollar cursos en formato CD-Rom, las nuevas tecnologías permiten optimizar los recursos de formación y han evolucionado hacia el nuevo WBT/IBT (WEb Based Training/Internet Based Training), valido en entornos Intranet o Internet. Actualmente, y aún que son tecnologías relativamente recientes, hay muchas opciones comerciales y gratuitas de WBT. Existen distintas recopilaciones y comparativas de plataformas de e-learning para proyectos formativos $^{5,6}$.

\section{Estándares}

Ante una disparidad de entornos, no interoperables, incompatibles, y sin ningún tipo de diseño para el desarrollo de estrategias comunes de implementación, la necesidad de establecer unos estándares en los programas on-line se convirtió en un requerimiento a pocos años de su aparición.

Aparecieron distintas iniciativas que buscaban la facilidad de uso de las nuevas tecnologías, su adaptación entre ellas, sus posibilidades de ampliación, ... otras centradas, básicamente, en el usuario que facilitaran la localización de los recursos educativos y protocolos de intercambio de datos, simplificando el intercambio entre distintos sistemas y estable- 
ciendo unas herramientas de comunicación entre ellas utilizables.

Actualmente, existen varios modelos de especificaciones y estándares de referencia internacional, los más conocidos y reconocidos son el AICC (Aviation Industry CPT Commitee) y el modelo SCORM ("Shareable Content Object Reference Model") ?.

$\mathrm{Su}$ finalidad es la de describir cuales son las especificaciones y estándares que se pueden utilizar, con el fin de crear materiales de aprendizaje, destinados a entornos web que sean reutilizables. El modelo SCORM, uno de los más extendidos y reconocidos en el ámbito de la formación, desarrollado por el Departamento de Defensa de los EUA, tenía y tiene por objetivo modernizar la educación y la formación por medio del uso de las nuevas tecnologías. Cuando, en la década de los noventa, se puso en marcha esta iniciativa, las tecnologías web ya estaban suficientemente desarrolladas para pensar en entornos virtuales en red diseñados al servicio de la formación.

Las especificaciones incluidas se resumen en cinco requisitos ${ }^{7}$ :

1. Accesibilidad: la capacidad de localizar y acceder a materiales de formación desde una ubicación remota y de distribuirlos en red.

2. Interoperatibilidad: la capacidad de utilizar los materiales en distintas plataformas y ubicaciones.

3. Durabilidad: la capacidad de resistir la evolución de la tecnologías sin que sea necesario recodificar o rediseñar a fondo los materiales.

4. Reutilizabilidad: la flexibilidad para incorporar los componentes formativos en distintos contextos y aplicaciones, como por ejemplo en diferentes actividades formativas.

5. Coste-efectividad: reducción de tiempo y de costos en la impartición de la formación.

Para conseguir estos requisitos, separa los materiales formativos de las plataformas (empaquetando los materiales en forma de ficheros de contenidos que los hagan reutilizables), etiqueta los materiales con metadatos para que sean posibles las búsquedas), y utiliza estándares (para poder asegurar la interoperabilidad y la durabilidad).

Estos metadatos hacen posible organizar los objetos de aprendizaje en bases de datos en red, los repositorios y por consiguiente localizar aquellos materiales que se correspondan con ciertos criterios: materia, tema, nivel educativo, requisitos de programa, etc.

Así mismo añade elementos adicionales, relativos a la interacción con el entorno virtual y concre- tamente sobre el control del recorrido del estudiante en el programa formativo posibilitando la obtención de información, por parte del participante a la actividad, del conocimiento de su proceso formativo y de los resultados obtenidos en cualquier momento del curso.

Actualmente, un consorcio de Academias, Gobiernos y organizaciones para la salud, están trabajando para desarrollar un modelo parecido al SCORM compilando especificaciones y estándares para la educación médica; este proyecto se llama MedBiquitous $^{8}$, y su finalidad es la de describir los estándares tecnológicos para mejorar la formación en las ciencias de la salud y conectar las organizaciones líderes en educación médica. Con estos estándares interoperables, los docentes tendrán un mejor acceso al intercambio de contenidos formativos pudiendo rastrear los recursos disponibles y construir una red de educación en ciencias de la salud más accesible, mesurable y efectiva ${ }^{8}$. Este proyecto, esta generando grandes expectativas en

\section{Tabla 1. Webs sobre indicadores de calidad en la formación on-line}

\section{Indicadores de éxito}

http://www.ihep.com

Indicadores de calidad de campus virtuales http://www.benvic.odl.org

Indicadores de calidad de formación on-line http://www.sav.us.es/pixelbit/articulos/n24

relación a la optimización de recursos científico-tecnológicos aplicados a la educación médica. (Tabla 1)

Accesibilidad

Los criterios de Accesibilidad universal, aceptados internacionalmente, están muy bien definidos en las normas WAI ${ }^{9}$ que pretenden llevar el desarrollo de la web (World Wide Web) a su máximo potencial, desarrollando tecnologías (especificaciones, directrices, software y herramientas). El W3C define la web como el universo de la red-accesible de la información (disponible a través de tu ordenador, telefono,...). Este universo posibilita nuevas formas de comunicación, ofreciendo oportunidades para compartir el conocimiento. Una de las principales metas del W3C es hacer que estos beneficios estén disponi- 
bles para todos, es decir ilustran un compromiso con el acceso universal. Los principios de simplicidad, modularidad, compatibilidad y extensibilidad son sus líneas básicas, así como la descentralización y la flexibilidad. En un sistema centralizado, todos los mensajes o acciones han de pasar por una autoridad central, causando embotellamientos cuando se incrementa el tráfico. La flexibilidad hace que la web no sea vulnerable y es una compañera indispensable de los sistemas distribuidos.

Es importante que las acciones de e-learning contemplen estos criterios, ya que van a constituir los pilares donde se soporte su estructura formativa.

Un buen programa educativo necesita facilidad de uso y navegación constantes. El concepto clave, en formación, sería la actualización y la atención continuada. El acceso del alumno a la actividad debe de ser rápido (ancho de banda y un tiempo de descarga de la página que no supere los diez segundos) e inmediato, es decir desde cualquier punto y siempre con los materiales actualizados. Asimismo, los procedimientos no deben ser complejos ya que si no, crean inseguridad al participante. Las dudas sobre determinados procesos y tareas deben de resolverse de forma rápida y concreta, de esta forma se mantiene la atención del alumno.

También se recomienda, para optimizar el espacio docente, que los scrolls sean horizontales y no verticales.

\section{Estructura del Programa.}

Calendario

Es importante que la estructuración del tiempo y la logística de un programa esté bien detallado, con tiempos previstos para cada actividad, que han de ser reales y proporcionales. Debe de definirse el calendario de distribución de materiales, la descripción logística del curso, y las informaciones complementarias que indiquen y faciliten el seguimiento del proceso formativo ${ }^{10}$.

Tiempo de dedicación

Además, el tiempo de dedicación requerido para el participante de una actividad docente ha de ser posible. En actividades de formación continuada, no se recomiendan más de unas cinco horas semanales de dedicación, ya que una actividad que requiera largas horas de dedicación diaria, puede considerarse en algunos casos inviable para el colectivo profesional en activo al que se oferta.

Aulas y tutores

Un elemento dinamizador y potenciador de la formación, es la organización de los participantes en gru- pos reducidos o aulas que permitan un relación más intensa y colaborativa entre los participantes. Estas aulas o grupos, deben ir dirigidos por un tutor (las funciones del cual describiremos en el apartado de metodología). Teniendo en cuenta que, en la formación a distancia, la interacción entre los participantes y las funciones del tutor tienen un papel clave y determinante para el éxito de la actividad docente y la obtención de resultados, es evidente que el número de alumnos asignados no puede ser excesivo (no se recomiendan más de 25-30 alumnos por aula virtual ${ }^{11}$.

\section{METODOLÓGICOS}

De la metodología educativa y de trabajo de los cursos de formación a distancia depende en gran medida el éxito del proceso de aprendizaje y la consecución de los objetivos propuestos.

La metodología es el factor clave de la formación a distancia, en ella todos los elementos que intervienen se interrelacionan: el papel del tutor, los contenidos, la comunicación, las herramientas disponibles, y se hacen necesarios entre ellos.

Los programas on-line han de buscar aquellos modelos que sirvan como entorno para facilitar el aprendizaje, las condiciones en las que se pueda aprender El conocimiento se entiende como dinámico, adquirirlo no supone memorizar una lista de elementos a reproducir en un examen, sino construir una comprensión propia de la materia. Es un proceso activo y colaborativo.

Teniendo en cuenta que esta formación va dirigida a profesionales de la salud en activo que necesitan actualizar, introducir nuevas técnicas o mejorar las utilizadas, es probable que este colectivo responda mejor a planteamientos prácticos tipo "learning by doing" que a exposiciones o provisiones de contenidos abstractos tradicionales.

Es conveniente que la metodología de trabajo incluya el desarrollo de actividades de producción, interacción y construcción colaborativa de nuevos conocimientos, exigiendo a los participantes una constante reflexión, análisis y crítica. Como abordan las nuevas teorías del aprendizaje ${ }^{12}$ es un proceso contextual. Aprendemos a partir de la experiencia, en la necesidad de reflexionar sobre la práctica. Sin reflexión no hay aprendizaje.

En este contexto, el blended learning ${ }^{13}$ sería uno de los formatos educativos más eficientes porque reúne lo mejor de la formación presencial y de las tecnologías. El tiempo y la experiencia han desembocado en la aparición de estos modelos llamados mixtos o modelos "blended" que combinan lo mejor de lo presencial con 
lo mejor de la virtualidad permitiendo dar respuesta a formaciones mucho más específicas ${ }^{14}$.

Tutor

Como conseguir este puente entre formación y práctica real, es donde el papel del tutor es determinante, por tanto es clave definir su papel.

Él es el responsable de definir claramente los requisitos y las horas de dedicación. También ha de ser concreto en las respuestas, ya que las evasivas desmotivan e inducen a la desconfianza del discente.

Un frecuente error es considerar que la formación a distancia significa formarse solo. Romper con esta falsa idea es una de las tareas fundamentales que debe asumir el tutor.

En una acción educativa "ideal", el tutor asume un papel dinamizador, contactando con el alumno durante todo el proceso de formación. Su misión es la de comentar, estimular y reforzar o introducir aquellos aspectos importantes a tener en cuenta.

También debe asumir la función de soporte. El tutor debe estar disponible para resolver problemas en relación con los contenidos estudiados.

Será responsabilidad del tutor (que debe ser un experto en los contenidos), introducir elementos dinamizadores, que despierten el interés y motiven al alumno, la participación, la asistencia facilitadora, la docencia, y el control sobre el aprendizaje.

Como experto desempeñará diversos roles:

1. Facilitador. Proporcionando un feed-back inmediato al alumno con el fin de captar su interés, atención y compromiso (prestando especial atención a la motivación). Orientando en las discusiones, y resolviendo dudas y problemas, ayudando a consolidar lo aprendido, y potenciando la reflexión conjunta.

2. Guía ("Coach"). Dirigiendo a los alumnos hacia los recursos más apropiados para la realización de las actividades.

3. Moderador. Gestionando las actividades y dinámicas de grupo en un entorno virtual.

Diseño de los contenidos.

Los contenidos educativos de los programas formativos a distancia, no pueden ser meras publicaciones con herramientas añadidas, sinó que deberían contemplar tres aspectos básicos:

1. Dimensión cognitiva. Fomentando el pensamiento crítico. Buscando los elementos que intervienen en la construcción del conocimiento, utilizando procesos de resolución de problemas en base a la: exploración, integración, resolución y aplicación.

2. Dimensión didáctica. Bien modulados y orientados.
Despertando el interés del participante. Introduciendo recursos que fomenten el aprendizaje. Es importante que el diseño llame la atención sobre los puntos de interés más relevantes, y que tengan una aplicación práctica. Buscando las actividades que faciliten el aprendizaje y la motivación del alumno.

3. Dimensión grupal. Fomentando la interactividad, debe de ser creativa y plural. Se incluirían todas aquellas acciones que promueven la dinámica grupal.

\section{Espacios de comunicación}

Uno de los factores clave del proceso de aprendizaje y del éxito en las actividades de formación a distancia es la comunicación entre profesor-estudiante y entre estudiante-estudiante.

En el e-learning, uno de los espacios de interés sobre procesos y resultados, tiene que ver con las oportunidades de comunicación temporal, ya sea sincrónica (al mismo tiempo) o asincrónica (en distintos tiempos, en diferido), unidireccional o bidireccional (Figura 1). Estos espacios proporcionan un entorno privilegiado para someter a valoración las acciones de e-learning, ya que nos posibilitan conocer como se producen las interacciones.

Resulta fundamental en el diseño de actividades, potenciar los elementos que faciliten la interacción/colaboración así como el papel de dinamización de las acciones del profesor-tutor y la dimensión temporal de su comunicación.

Figura 1. Actividades según tipos de comunicación

\begin{tabular}{|c|c|c|}
\hline \multirow{2}{*}{$\begin{array}{c}\text { Tipos de } \\
\text { comunicación }\end{array}$} & \multicolumn{2}{|c|}{ DIMENSIÓN TEMPORAL } \\
\hline & SINCRONA & ASSINCRONA \\
\hline UNIDIRECCIONAL & $\begin{array}{l}\text { Audio Conferencia } \\
\text { Videoconferencia } \\
\text { Seminarios en línea } \\
\text { (Webminars) }\end{array}$ & $\begin{array}{l}\text { Grabaciones en vídeo } \\
\text { o audio } \\
\text { Tablones de anuncios } \\
\text { Hipertextos } \\
\text { Newgroups } \\
\text { Envío de Ficheros } \\
\text { Listas de distribución } \\
\text { Calendario/Agenda }\end{array}$ \\
\hline $\begin{array}{c}\text { BI/MULTI } \\
\text { DIRECCIONAL }\end{array}$ & $\begin{array}{l}\text { Discusiones en vídeo } \\
\text { en tiempo real } \\
\text { Chat/messenger } \\
\text { Software para compartir } \\
\text { aplicaciones } \\
\text { Pizarras/Escritorios } \\
\text { compartidos } \\
\text { Aplicaciones de } \\
\text { realimentación inmediata: } \\
\text { votaciones, encuestas, } \\
\text { autoevaluaciones. }\end{array}$ & $\begin{array}{l}\text { Tablones de anuncios } \\
\text { electrónicos } \\
\text { E-mail } \\
\text { Foros de discusión } \\
\text { electrónicos }\end{array}$ \\
\hline
\end{tabular}


Para que los medios de comunicación sean efectivos, es importante que existan diferentes tipos de interacción, y espacios con distintos niveles de formalidad alguna moderada y dirigida y otros no, que permitan el desarrollo de una gama de distintos niveles de interacción entre los participantes y de intensidades, desde papeles centrales a papeles localizados en la periferia del aula, grupo, o comunidad de aprendizaje.

Herramientas para crear actividades

Existen distintas herramientas para crear actividades de contenido, como: vídeos, diapositivas, cámaras, archivos de audio, combinación de vídeo y diapositivas narradas de PowerPoint, transferencia de vídeo clip desde casete o cámara a CDRom, vídeos de captura de pantalla, técnicas de cómputo, envíos de ficheros, newsgroups, .... y otras de interacción, como: chats, foros, simuladores, escritorios compartidos, voz IP,...

Algunas de estas herramientas de interacción, como los foros, representan una oportunidad para que los docentes dirijan el aprendizaje de los alumnos, proporcionándoles la posibilidad de introducir múltiples actividades. En ellos, los profesores/alumnos interaccionan, formulan y contestan preguntas, expo-

\section{Tabla 2. Recursos útiles de Educación Médica en Internet}

\begin{tabular}{|c|c|c|c|}
\hline Tipología & Institución & Dirección URL & Características \\
\hline \multirow[t]{3}{*}{$\begin{array}{l}\text { Formación Médica } \\
\text { Continuada }\end{array}$} & Online Continuing Medical Education & http.//www.cmelist.com & $\begin{array}{l}\text { Listado de actividades on-line } \\
\text { clasificadas por especialidades (USA) }\end{array}$ \\
\hline & Stanford School of Medicine & http.//cme.stanford.edu & $\begin{array}{l}\text { Actividades a distancia en distintas } \\
\text { especialidades }\end{array}$ \\
\hline & $\begin{array}{l}\text { American Academy of Family Physicians } \\
\text { The Oncologist CME Online } \\
\text { E-learning Europa } \\
\text { Toute la Formation Médicale continue } \\
\text { (TLM-Online) }\end{array}$ & $\begin{array}{l}\text { http.//www.aafp.org/online } \\
\text { http.//cme.alphamedpress.org } \\
\text { http://elearningeuropa.info } \\
\text { http://www.tlmfmc.com/site/online.tpl }\end{array}$ & $\begin{array}{l}\text { Medicina de Familia } \\
\text { Oncología } \\
\text { Radiología } \\
\text { Listado de actividades on-line } \\
\text { clasificadas por especialidades (EU) }\end{array}$ \\
\hline \multirow[t]{5}{*}{$\begin{array}{l}\text { Organizaciones } \\
\text { de Educación Médica }\end{array}$} & $\begin{array}{l}\text { Association of American Medical } \\
\text { Colleges /AAMC) }\end{array}$ & http.//www.aamc.org/meded/mededportal & $\begin{array}{l}\text { Repositorio } \\
\text { Banco virtual de pacientes } \\
\text { Material relacionado } \\
\text { con la educación de competencias } \\
\text { "peer reviewed" }\end{array}$ \\
\hline & $\begin{array}{l}\text { Palliative Education Resource Center } \\
\text { (EPERC) }\end{array}$ & http.//www.eperc.mcw.edu & $\begin{array}{l}\text { Repositorio } \\
\text { Publicaciones digitales } \\
\text { Links con otros recursos on-line } \\
\text { "peer reviewed" }\end{array}$ \\
\hline & $\begin{array}{l}\text { The Health Education Assets Library } \\
\text { (HEAL) }\end{array}$ & http.//www.healcentral.org & $\begin{array}{l}\text { Repositorio } \\
\text { Publicaciones digitales } \\
\text { Recursos para el aprendizaje } \\
\text { "peer reviewed" }\end{array}$ \\
\hline & $\begin{array}{l}\text { Multimedia Educational Resource for } \\
\text { Learning and Online Teaching (MERLOT) }\end{array}$ & http.//www.merlot.org & $\begin{array}{l}\text { Repositorio } \\
\text { Links con otros recursos on-line } \\
\text { pero con "peer reviewed } \\
\text { Sección de Tecnología }\end{array}$ \\
\hline & $\begin{array}{l}\text { International Virtual Medical School } \\
\text { (IVIMEDS) }\end{array}$ & http.//www.ivimeds.org & $\begin{array}{l}\text { Repositorio (solo para asociados) } \\
\text { Establecimiento de estándares } \\
\text { en Educación Médica } \\
\text { Formación Mixta (Blended learning) }\end{array}$ \\
\hline \multirow[t]{2}{*}{$\begin{array}{l}\text { Revistas Médicas } \\
\text { Electrónicas }\end{array}$} & EMedicine & http://www.emedicine.com & $\begin{array}{l}\text { Repositorio } \\
\text { Revistas electrónicas } \\
\text { Formación on-line }\end{array}$ \\
\hline & MEDLINE/PubMed & http://www.nlm.nih.gov/ & $\begin{array}{l}\text { Dispone de } 4600 \text { revistas en Formato } \\
\text { electrónico con más de } 12 \text { millones } \\
\text { de referencias }\end{array}$ \\
\hline Atlas Médicos & The Visible Human Project & http://www.nlm.nih.gov/onlineexhibitions.htm & Atlas de anatomía virtual y simulaciones \\
\hline Ensayos clínicos & $\begin{array}{l}\text { US Department of Health and Human } \\
\text { Services }\end{array}$ & http://www.fda.gov & $\begin{array}{l}\text { Información sobre ensayos clínicos, en } \\
\text { especial sobre Endocrinología y Nutrición }\end{array}$ \\
\hline $\begin{array}{l}\text { Guías de práctica } \\
\text { clínica }\end{array}$ & WebMD & http://www.healtheon.com & Salud Pública \\
\hline
\end{tabular}


Figura 2. Instrumentos de evaluación del aprendizaje

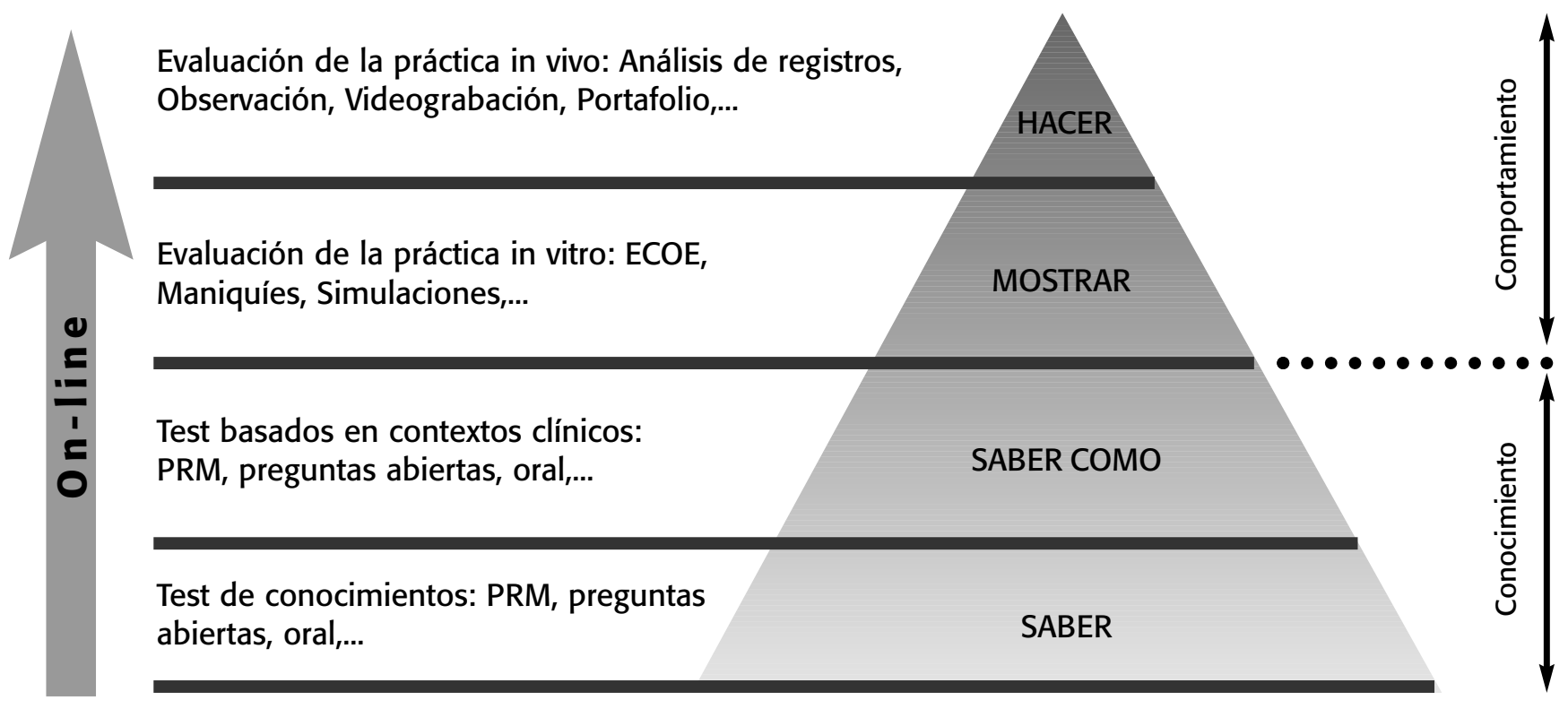

Fuente: Cees van der Vleuten • University of Maastricht • "A paradigm shift in education: How to proceed with assessment?" 9th International Ottawa Conference on Medical Education • Cape Town, 28 February - 3 March 2000. (modificada)

nen ideas, realizan debates, resuelven casos, desarrollan proyectos, y solucionan problemas (Tabla 2).

Otras, como las simulaciones, se reafirman como una modalidad emergente de futuro ya que con el desarrollo tecnológico pueden hacer más énfasis educativo en las habilidades y los razonamientos clínicos. Las simulaciones intentan reproducir situaciones lo más reales posibles, ofrecen una serie de ventajas y aseguran la seguridad de los pacientes. Este tipo de formación, muy efectiva, ya se ha institucionalizado en muchas otras profesiones de alto riesgo (aviación, militar, nuclear,..) y la educación médica tiene que apostar por las ventajas que representa.

Existen varios tipos de simulaciones, a grandes rasgos podrían agruparse en dos tipologías:

Simulaciones blandas: conversación con personajes virtuales en pantalla; de gestión y toma de decisiones en distintos escenarios; de gestión analítica que ayudan a interpretar datos y también otros basados en problemas.

Simulaciones duras: toma de decisiones prágmaticas y de respuesta en situaciones de emergencia; de diagnostico; de resolución de casos, y también de cirugía u otras especialidades técnicas

Este tipo de actividades ofrecen una serie de ventajas educativas, ya que ofrecen seguridad ante los errores en entrenamientos de técnicas de alto riesgo, ofrecen un coste más reducido y menos peligroso que trabajar con la realidad o también pueden ayudar a la compresión de procesos.

Aún así, también existen una serie de desventajas relacionadas con el coste, su dificultad de adaptación a los entornos reales, las dificultades que generan el aprendizaje del uso de las herramientas propias de la simulación y su desarrollo.

En el mercado existen servicios para crear simulaciones por encargo pero tienen una difícil rentabilidad. También existen modelos preconstruidos, algunos de los cuales, pueden modificarse para adaptarse a las necesidades del usuario, así como algunas herramientas que permiten al usuario desarrollar sus propias simulaciones.

\section{EVALUATIVOS}

La evaluación de las actividades formativas nos permiten revisar los procedimientos, elaborar planos de mejora, beneficiar los resultados y las posteriores ediciones de las actividades formativas.

La evaluación formativa tiene un impacto educativo muy importante que orienta y refuerza el aprendizaje ${ }^{15}$. Su finalidad ha de ser metodológica, para guiar, orientar y ayudar a través del proceso de maduración y formación, proporciona un abundante feed-back sobre la propia práctica profesional y ofrece la posibilidad al participante de convertirse el mismo, en objeto de análisis ${ }^{16}$. El formato de la evaluación, esconde un elemento subyacente que dirige el proceso formativo, ya que, frecuentemente, los 
estudiantes adaptarán su estudio al tipo de formato evaluativo que se utilice (Figura 2).

Básicamente, encontramos 3 tipologías evaluativas:

De contenidos. Desarrollan estrategias de aprendizaje teóricas. Estas evaluaciones se practican de manera descontextualizada y miden principalmente la memorización de hechos (test de conocimientos).

De aprendizaje. Pretenden conocer la relación entre los contenidos y las actitudes, plantean estrategias de aprendizaje reflexivas con preguntas abiertas que potencian la síntesis de los conocimientos adquiridos. Un ejemplo serían casos clínicos compuestos por preguntas contextualizadas, a partir de una situación clínica. (tests basados en contextos clínicos)

De práctica. Buscan la aplicación de conocimientos en situaciones simuladas, potencian estrategias pragmáticas de puesta en funcionamiento de los elementos que han intervenido en la actividad docente. Es un tipo de evaluación mucho más compleja, que intenta establecer un puente entre formación y práctica. (evaluación de la práctica "in vitro" o "in vivo").

El aprendizaje es un proceso formativo-madurativo. No es suficiente conocer los contenidos, sino que hay que saber aplicarlos cuando corresponda. La calidad en la evaluación se interrelaciona en función de la práctica real y su evolución.

La formación a distancia debe dirigir el aprendizaje permitiendo ensayar esta práctica "in vitro" mientras se adquieren los conocimientos, para poder llegar con los menores riesgos posibles a la práctica "in vivo".

\section{BIBLIOGRAFÍA}

1. Declaración de Bolonia.. Dipòsit Digital de Documents de la UAB - Servei de BibliotequesDipòsit Digital de Documents de la UAB - Servei de Biblioteques http://ddd.uab.es/?ln=ca

2. Kearney, N; Comunidades de aprendizaje: un enfoque pedagógico de futuro, 2002. Proyecto Prometeus. [Fecha de consulta 7/03/2005] www.prometeus.org

3. Wenger, Etienne, Themes an Ideas: Communities of Practice, $2002 \mathrm{http}: / /$ www.ewenger.com/ewthemes.html

4. Wutoh, R; et al., eLearning: A review of Internet-Based Continuing Medical Education. The Journal of Continuing Education in the Health Professions, Volume 24, pp.20-30. USA 2004

5. http://e-learning.bankhacker.com

6. http://ww.gate.upm.es

7. SCORM. http://www.scorm.com

8. MedBiquitous. http://www.medbiq.org

9. World Wide Web. http://www.w3c.org

10. Pardell, H., director. Formación continuada a distancia. Barcelona. Mayo, SA, 2004.

11. Pincas, A. "Gradual and Simple changes to incorporate ICT into de Classroom" En elearningeuropea.info. [Revista en línea] [Fecha de consulta 7/09/2005] http://www.elearningeuropa.info/doc

12. Schön, DA. "The reflective practitioner. New Cork: Basic Books; 1983

13. Bartolome, A. "Blended Learning. Conceptos básicos". En Revista PIXEL_BIT. Monográfico de Blended Learnming [Revista en línea] [Fecha de consulta 7/07/2006] nº 23 http://www.imi.ub.es/personal/bartolome/articuloshtmlmultimedia_94

14. Brennan. M. "Blended Learning and Business Change". Chief Learning Officer Magazine. [Revista en línea] [Fecha de consulta 20/06/2006] http://www.clomedia.com/content

15. Garrison, R. et al., Critical Thinking and Computer Conferencing : A Model and Tool to Asses Cognitive Presence, 2002 [Fecha de consulta 3/03/2003] http://www.atl. ualberta.ca/cmc/CogPresPaper_June30_.pdf

16. Newman, DR. et al., An experiment in group learning technology: evaluating critical thinking in face-to-face and computer supported seminars. Queen's University, Belfast, 1996 http://qub.ac.uk 